\title{
Adverse events and survival after closing- and opening-wedge high tibial osteotomy: a comparative study of 412 patients
}

\author{
T. Duivenvoorden ${ }^{1} \cdot$ P. van Diggele ${ }^{1} \cdot$ M. Reijman ${ }^{1} \cdot$ P. K. Bos ${ }^{1} \cdot$ J. van Egmond $^{1} \cdot$ \\ S. M. A. Bierma-Zeinstra ${ }^{1,2}$ J. A. N. Verhaar ${ }^{1}$
}

Received: 1 October 2014 / Accepted: 8 May 2015 / Published online: 31 May 2015

(C) The Author(s) 2015. This article is published with open access at Springerlink.com

\begin{abstract}
Purpose Varus medial knee osteoarthritis (OA) can be treated with a closing-wedge $(\mathrm{CW})$ or opening-wedge (OW) high tibial osteotomy (HTO). Little is known about the adverse event (AE) rate of these techniques. The purpose of this study was to examine the $\mathrm{AE}$ rate and survival rate of a consecutive series of 412 patients undergoing CWor OW-HTO.

Methods Medical records were retrospectively screened, and all patients who underwent HTO from 1993 to 2012 at the Erasmus University Medical Centre were assessed with a self-administered questionnaire. Patients filled in the intermittent and constant osteoarthritis pain score, knee injury and osteoarthritis outcome score, and a general questionnaire focusing on AE.
\end{abstract}

T. Duivenvoorden

t.duivenvoorden@erasmusmc.nl

P. van Diggele

p.vandiggele@erasmusmc.nl

M. Reijman

m.reijman@erasmusmc.nl

P. K. Bos

p.k.bos@erasmusmc.nl

J. van Egmond

j.vanegmond@erasmusmc.nl

S. M. A. Bierma-Zeinstra

s.bierma-zeinstra@erasmusmc.nl

J. A. N. Verhaar

j.verhaar@erasmusmc.nl

1 Department of Orthopaedics, Erasmus University Medical Centre, PO Box 2040, 3000 CA Rotterdam, The Netherlands

2 Department of General Practice, Erasmus University Medical Centre, Rotterdam, The Netherlands
Results Medical records of 412 patients (354 CWand 112 OW-HTOs) were screened. Of the 358 eligible patients, $291(81 \%)$ returned their questionnaire. A total of $80 \mathrm{AE}(17 \%)$ were found in 466 osteotomies. In the CW-group, $47(13 \%)$ serious adverse events (SAE) and 2 $(0.6 \%)$ AE were found. In the OW-group, 17 (15\%) SAE and $14(13 \%) \mathrm{AE}$ were found. The most common AE was in $14(4 \%)$ patients of the CW-group sensory palsy of the common peroneal nerve. The most common $\mathrm{AE}$ in the OW-group was persistent pain at the iliac crest [11 (9.8\%) patients]. Hardware was removed in $48 \%$ of the CWosteotomies and $71 \%$ of the OW-osteotomies $(p<0.05)$. The probability of survival was $75 \%$ after 10 years in the CW-group versus $90 \%$ in the OW-group $(p<0.05)$. In both groups, an equal number of patients were "in need for prosthesis" according to OARSI criteria.

Conclusion OW-HTO was associated with more AE than CW-HTO. OW-HTO resulted in better survival than CWHTO. However, in both groups an equal number of patients were in need for prosthesis.

Level of evidence Retrospective comparative study, Level III.

Keywords Opening-wedge high tibial osteotomy . Closing-wedge high tibial osteotomy $\cdot$ Adverse events . Survival

\section{Introduction}

Knee osteoarthritis (OA) is one of the most common joint disorders and causes considerable pain and immobility. In case of varus alignment, the medial compartment is mostly affected $[1,17]$. Varus medial knee OA in young and active patients can be treated with a valgus high tibial osteotomy 
(HTO). Various techniques are available among which closing-wedge $(\mathrm{CW})$ and opening-wedge (OW) HTO are performed most frequently. Both have advantages and disadvantages, and overall good short- and midterm outcomes have been reported $[2,5,7,12,14,21]$.

Although types of adverse events after HTO are well described, little is known about their actual incidence [3, $5,15,18,19]$. In a prospective study of 40 patients, Van Bekerom et al. found significant more adverse events in an OW-group, whereas in a randomized controlled trial of 50 patients, Gaasbeek et al. found a higher adverse event rate in a CW-group. Song et al. found no difference in adverse events rate in their retrospective study of 194 patients [8, $18,19]$. Thus, only a few relatively small studies have compared the adverse event rate of CW- and OW-HTO, and the results of these studies are contradictory.

The success of a HTO is expressed in the number of years until conversion to a joint prosthesis is performed. Several studies have studied the survival of the HTO and factors influencing the survival $[6,12,21]$. They all defined failure as redo procedure of the HTO or conversion to unicompartmental knee arthroplasty (UKA) or total knee arthroplasty (TKA). However, this end-point may introduce a decision bias and thus lead to an overestimation of the survival, because the decision to convert an HTO is affected by the opinion of patient as well as surgeon. Patients who do not undergo further surgery do not necessarily have a good result and might have high pain scores and a low functional outcome. Therefore, the Osteoarthritis Research Society International (OARSI) defined criteria for a surrogate measure of the "need for joint replacement surgery" [9]. With these criteria, it is possible to define a nonsurvivor based on the pain score and functional outcome. Adjustment of the original survival rate with this patientreported outcome-based measure would result in a more accurate estimate of the real survival.

The hypothesis of this retrospective study was to find a higher adverse event rate and lower survival rate in patients undergoing OW-HTO in comparison with CW-HTO.

\section{Materials and methods}

The medical records of all patients who underwent CW- or OW-HTO at the [Erasmus Medical Centre] (a university teaching hospital) between 1993 and 2012 were screened. This period was chosen for the reason that medical records are preserved for at least 20 years in our hospital and we would achieve a minimal follow-up of 1 year.

All 412 patients were asked to fill in a written questionnaire in 2013. When patients did not respond within 3 weeks, they were contacted by telephone. When these patients did not answer their telephone, a reminder was sent by mail after checking their address in the municipal administration.

\section{Measurements}

Patient characteristics such as gender, preoperative age, body mass index (BMI) and hip-knee-ankle angle (HKA angle) were collected for all 412 patients. Medical records were screened to identify the osteotomy technique, operating time, type of fixation material and adverse events. Pain, functional status and adverse events were measured with patient-reported outcome measures (PROMs). Pain and functional status were measured to define a survivor according to the OARSI criteria [9].

Pain was measured with the intermittent and constant osteoarthritis pain (ICOAP) score (0-100) [10]. The functional status was measured with the Knee injury and Osteoarthritis Outcome Score-Physical Function Short Form (KOOS-PS) [3, 4, 16]. The KOOS-PS is intended to elicit people's opinions about the difficulties they experience with activity due to problems with their knee. Standardized response options are given (5-point Likert scale), and each question was scored from 0 to 4 . Then, a normalized score from 0 to 100 is calculated. The criteria defined by the OARSI to determine patients in need for joint replacement surgery were used. They concluded that the sum score [pain (measured with the ICOAP (0-100)) + physical function (measured with the KOOS-PS $(0-100))>80$ ] is a discriminatory cut-off point to define an indication for joint replacement [9].

\section{Adverse events}

Adverse events were initially assessed from medical records. Moreover, to avert under-registration, adverse events were also assessed with a self-administered questionnaire at follow-up. Patients were specifically asked in this questionnaire for wound infection, thromboembolism, bleeding, paraesthesia, dropping foot, reflex sympathetic dystrophy syndrome, persistent pain or pain at the iliac crest, non-union. When patients scored positive for an adverse event, the adverse event was checked by a telephonic examination. In this study, the adverse events of all 412 patients are presented, of whom the medical records were screened.

Adverse events were classified into adverse events (AE) and serious adverse events (SAE). Adverse events were defined as serious when patients have an undesirable experience associated with a medical intervention leading to death, a life-threatening situation, initial or prolonged hospitalization, disability or permanent damage, or a needed intervention to prevent permanent impairment or damage according to the definition of the FDA. 
Table 1 Patient characteristics of the total study population, separately for opening-wedge versus closing-wedge HTO and responders versus non-responders

\begin{tabular}{|c|c|c|c|c|c|}
\hline & $\begin{array}{l}\text { Total group of } \\
\text { osteotomies }(n=466)\end{array}$ & $\begin{array}{l}\text { Closing-wedge } \\
\text { osteotomy }(n=354)\end{array}$ & $\begin{array}{l}\text { Opening-wedge } \\
\text { osteotomy }(n=112)\end{array}$ & $\begin{array}{l}\text { Responders } \\
(n=291)\end{array}$ & $\begin{array}{l}\text { Non-responders } \\
(n=121)^{\mathrm{a}}\end{array}$ \\
\hline Follow-up time (years) & $9.8(4.9)$ & $10.6(5.1)^{\dagger}$ & $7.4(3.2)^{\dagger}$ & NA & NA \\
\hline Women, $n(\%)$ & $190(40.8)$ & $151(42.7)$ & $39(34.8)$ & $134(46)$ & $36(30)$ \\
\hline Age $(y r s)^{b}$ & $49.2(9.3)$ & $49.4(9.0)$ & $48.7(10.1)$ & $49.7(8.7)$ & $47.9(10.6)$ \\
\hline BMI $\left(\mathrm{kg} / \mathrm{m}^{2}\right)^{\mathrm{b}}$ & $29.1(5.4)$ & $29.5(5.8)$ & $28.5(4.5)$ & $29.0(5.4)$ & $29.3(5.3)$ \\
\hline HKA angle $\left({ }^{\circ}\right)^{\mathrm{b}, \mathrm{c}}$ & $6.6(2.6)$ & $6.3(2.2)^{\dagger}$ & $7.4(3.5)^{\dagger}$ & $6.6(2.6)$ & $6.6(2.7)$ \\
\hline Surgery time (min) & $116.9(30.3)$ & $112.7(28.8)^{\dagger}$ & $130.3(31.4)^{\dagger}$ & $118.8(30.7)$ & $112.2(29.1)$ \\
\hline $\begin{array}{l}\text { Duration of hospitaliza- } \\
\text { tion (days) }\end{array}$ & $5.5(2.9)$ & $5.5(2.3)$ & $5.5(4.2)$ & $5.2(2.4)$ & $6.1(3.6)$ \\
\hline
\end{tabular}

All values are presented as mean $( \pm \mathrm{SD})$ unless stated otherwise

$B M I$ body mass index, deg degree, HTO high tibial osteotomy, HKA angle hip-knee-ankle angle, min minutes, NA not applicable, yrs years

${ }^{\dagger} p<0.05$ for difference between the two groups

a $77 \%$ of the lost patients underwent closing-wedge HTO

b The preoperative values are presented

c A positive value means varus malalignment

\section{Surgical techniques}

\section{Closing-wedge group}

CW-HTO was performed with a lateral approach. The common peroneal nerve (CPN) was exposed and retracted. The proximal tibiofibular joint was opened, and $1 \mathrm{~cm}$ of the proximal tibiofibular bone was resected. The proximal osteotomy site and the slope of the osteotomy were marked using two Kirschner (K)-wires. Under C-arm guidance, the first osteotomy was performed with an oscillating saw and completed with an osteotome. The second osteotomy was performed with help of an aiming device (Arthrex, Naples, Florida). Fixation was achieved using two staples or a Tomofix plate. Different types of fixation material were used over years; staples (Stryker, Schönkirchen, Germany), Tomofix plate (Synthes GmbH, Oberdorf, Switzerland) and Puddu plate (Arthrex, Naples, Florida). Before closing the wound, a fasciotomy of the anterior compartment was performed.

\section{Opening-wedge group}

OW-HTO was performed with an anteromedial incision. The pes anserinus tendons and medial collateral ligament were dorsally retracted. Under C-arm guidance, two $\mathrm{K}$-wires were placed in an oblique fashion from medial to lateral to serve as a cutting guide for the osteotomy. An osteotomy was performed using an oscillating saw. Care was taken to avoid violation of the lateral cortex. The distracting osteotomes were placed ventrally and dorsally and gradually distracted to achieve the right alignment.
OW-osteotomies were fixated using either a Puddu plate without plate locking screws (until 2006) or Tomofix plate (from 2006 until now). In 56 patients with large wedges of the OW-group, a spongioplasty was performed using autologous bone harvested at the iliac crest. The Ethics Committee of the Erasmus Medical Centre approved the protocol (MEC-2013-140).

\section{Statistical analysis}

Statistical analysis was performed using PASW statistics version 20 (SPSS science Inc., Chicago, USA), and a $p$ value $<0.05$ was considered as statistically significant. Data of CW- and OW-HTO patients are presented separately. Between-group differences were tested with the independent $t$ test (Student's $t$ test) or $\chi^{2}$ test.

To evaluate the presence of a possible selective dropout during follow-up, baseline characteristics of the responders (those who filled in the questionnaire) were compared with the non-responders using the Kruskal-Wallis or $\chi^{2}$ test.

Differences between CW- and OW-HTO patients were analysed using the independent $t$ test (Student's $t$ test) or $\chi^{2}$ test.

Multiple survival analyses according to Kaplan and Meier were carried out. In the first survival analysis, conversion to a UKA or TKA was considered as end-point. In the second survival analysis, "being in need for a UKA or TKA" according to the OARSI criteria was considered as end-point [13]. Patients with a conversion to a UKA or TKA were also considered as "being in need for a UKA or TKA". Differences in survival between CW- and OW-HTO were calculated using the log rank test (Table 1). 


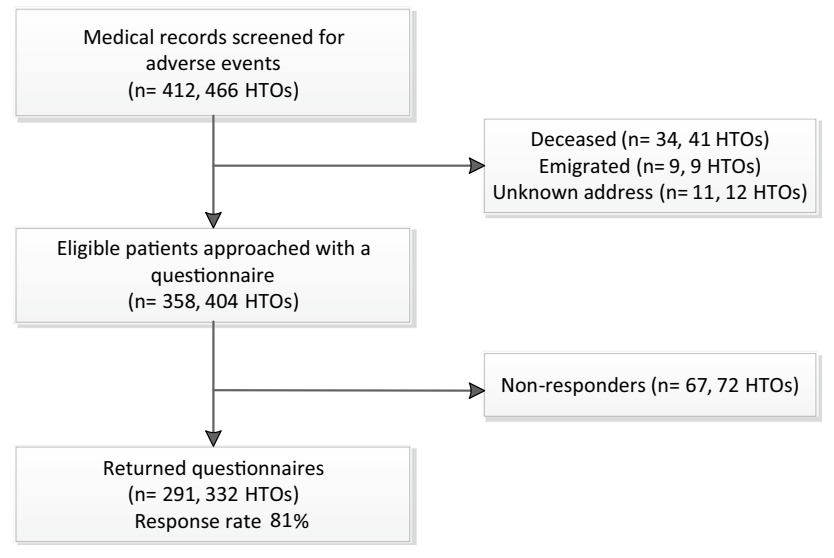

Fig. 1 Flowchart of the study. HTO high tibial osteotomy

We have found in this study a RR of 2.0 with $49 / 354$ $\mathrm{AE}$ in the CW-group and 31/112 AE in the OW-group. The power of this study with 354 patients in the CW-group and 112 patients in the OW-group was 0.94 to find a RR of 2.0. Concerning the survival after HTO, we found in this study a RR of 3.0 with 73/354 non-survivors in the CW-group and $8 / 112$ in the OW-group. The power of this study with 354 patients in the CW-group and 112 patients in the OWgroup was 0.99 to find a RR of 3.0 (Fig. 1).

\section{Results}

\section{Adverse events}

A total of $80 \mathrm{AE}(17 \%)$ were found in 466 osteotomies. In the CW-group, $47(13 \%) \mathrm{SAE}$ and $2(0.6 \%) \mathrm{AE}$ were found. In the OW-group, 17 (15\%) SAE and $14(13 \%) \mathrm{AE}$ were found. The most common AE was in $14(4 \%)$ patients of the $\mathrm{CW}$-group temporary sensory palsy of the CPN. In the OW-group, the most common AE persistent pain was in $11(19.7 \%)$ patients at the iliac crest of those who had cancellous bone grafting from the iliac crest in OW-HTO. Moreover, a non-union occurred in 12 patients $(8$ (2.3\%) in the CW-group and 4 (3.6\%) in the OW-group). Hardware was removed in $48 \%$ of the CW-osteotomies and $71 \%$ of the OW-osteotomies $(p<0.05)$. All adverse events are outlined in Table 2.

\section{Survival}

During the follow-up period, 81 osteotomies $(17.4 \%)$ have been revised to UKA or TKA: 73 in the CW-group and eight in the OW-group (Fig. 3). When conversion to UKA or TKA was considered as end-point, the OW-group had a better survival than the CW-group ( $p<0.05$; Fig. 2 ). When the OARSI criteria for "being in need for a UKA or TKA"

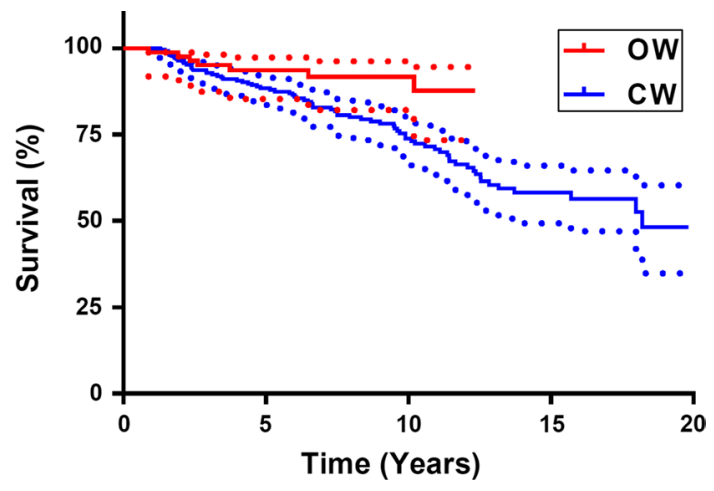

Fig. 2 Survival curve of closing- and opening-wedge osteotomy. Survival considered with conversion to UKA or TKA as end-point

was considered as end-point and this was added to conversion to a prosthesis, no difference in survival between CWand the OW-group was found.

\section{Discussion}

The aim of this study was to present the adverse event rate and survival rate of 466 HTOs performed in our university teaching hospital. The most important finding of this study was the overall adverse event rate of $28 \%$ in the OWgroup and $14 \%$ in the $\mathrm{CW}$-group. This $\mathrm{AE}$ rate is lower than reported in several studies. Other studies show adverse event rates ranging from 24 to $63 \%[2,13,18,19]$. However, Gaasbeek et al. reported an adverse event rate of $12 \%$ [8]. The higher adverse event rate in the OW-group is in agreement with Van Bekerom et al. who reported $55 \%$ adverse events in the OW-group $(n=20)$ and $20 \%$ in the CW-group $(n=20)$. However, Song et al. found more adverse events in the CW-group ( $28 \%, n=104)$ than in the OW-group $(20 \%, n=90)$. Miller et al. and Floerkemeier et al. studied only adverse events after OW-osteotomy. They reported adverse event rates of $37 \%(n=46)$ and $6 \%(n=533)$, respectively $[7,15]$. Results of the different studies seem to be contradictory. In the majority of the studies, only medical records were screened to identify adverse events. This could have led to an underestimation. The strength of this study is the additional information assessed with a self-administered questionnaire after screening of the medical records in a relatively large number of patients. Consequently, we assume that our results are more accurate estimate of the real adverse event rate.

Different types of fixation material were used over years; however, no difference was found in removal rate between staples and plates. The hardware removal rates are similar to those in the literature. Multiple studies report a hardware removal rate of $>50 \%$ of the patients in an OW-group and 
Table 2 Adverse events for the closing- and opening-wedge group

\begin{tabular}{|c|c|c|}
\hline & \multicolumn{2}{|l|}{ Number of events, $n(\%)$} \\
\hline & Closing-wedge osteotomy $(n=354)$ & Opening-wedge osteotomy $(n=112)$ \\
\hline \multicolumn{3}{|l|}{ Serious adverse events } \\
\hline Sensory palsy of the CPN & $14(4.0)$ & 0 \\
\hline Motor palsy of the CPN & $1(0.3)$ & 0 \\
\hline Pseudoarthrosis & $8(2.3)$ & $4(3.6)$ \\
\hline Wound infection treated with antibiotics & $6(1.7)$ & $5(4.5)$ \\
\hline Fracture of the tibial plateau & $2(0.6)$ & $2(1.9)$ \\
\hline Re-HTOa & $7(2.0)$ & $3(2.7)$ \\
\hline Delayed union & $1(0.3)$ & 0 \\
\hline Lesion of the ATA & $1(0.3)$ & 0 \\
\hline Malposition of hardware & $1(0.3)$ & 0 \\
\hline Deep venous thrombosis & $2(0.6)$ & 0 \\
\hline Pulmonary embolus & 0 & $1(0.9)$ \\
\hline Infection of the urinary tract & $2(0.6)$ & $1(0.9)$ \\
\hline Post-surgery diffuse lung emphysema & $1(0.3)$ & 0 \\
\hline Compartment syndrome & $1(0.3)$ & $1(0.9)$ \\
\hline Hardware removal $^{\mathrm{b}}$ & $169(47.7)$ & $79(70.5)$ \\
\hline \multicolumn{3}{|l|}{ Adverse events } \\
\hline Iliac crest pain & 0 & $11(19.7)^{\mathrm{c}}$ \\
\hline Wound infection without antibiotic treatment & $1(0.3)$ & $2(1.9)$ \\
\hline CRPS & $1(0.3)$ & $1(0.9)$ \\
\hline
\end{tabular}

One hundred and twenty patients did not return their questionnaire for several reasons; their adverse events were only assessed by medical record screening

$C P N$ common peroneal nerve, ATA anterior tibial artery, CRPS complex regional pain syndrome

${ }^{\text {a }} \mathrm{Re}$-HTO was performed because of overcorrection or undercorrection or loss of correction

b Ten hardware removals in the closing-wedge group and two in the opening-wedge group were performed prior to total knee arthroplasty

${ }^{c}$ Fifty-six patients $(50 \%)$ of the opening-wedge group underwent spongioplasty with autologous bone harvested at the iliac crest. Of these patients, 11 patients reported pain at the iliac crest for more than 6 weeks

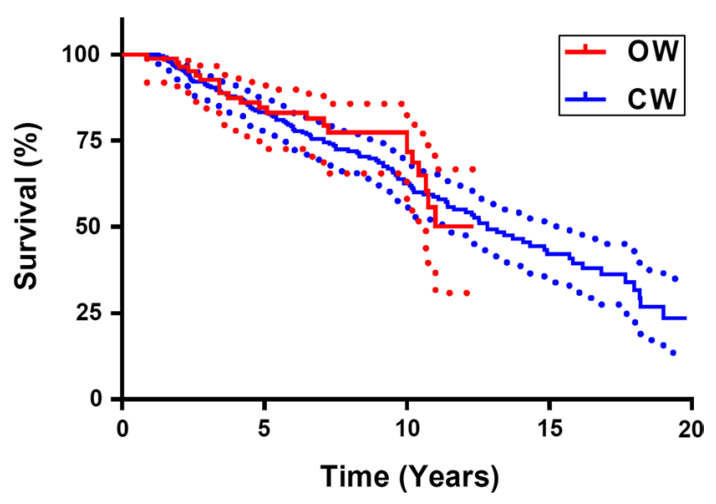

Fig. 3 Survival curve of closing- and opening-wedge osteotomy. Survival considered with "being in need for a UKA of TKA" according to the OARSI criteria in addition to "being conversed to UKA or TKA" as end-point

significantly more hardware removals in the OW-group in comparison with the CW-group [5, 11, 20]. The more superficial medial position of hardware with less coverage of soft tissue might be a possible explanation for the higher hardware removal rate in the OW-group.

Another major adverse event was pain at the iliac crest, caused by harvesting the cancellous bone for gap filling in an OW-HTO. In a recent randomized controlled trial, Zorzi and colleagues concluded that a cancellous bone graft in wedges $<12.5 \mathrm{~mm}$ is not necessary [22]. It is clear that the complication rate will decrease in the OW-group when bone grafting can be avoided.

During the follow-up period, $17.4 \%$ of the osteotomies have been revised to UKA or TKA. When conversion to prosthesis was taken as end-point, the OW-group had a significantly better survival than the $\mathrm{CW}$-group. For CW-osteotomy, the probability of survival was $75 \%$ after 10 years and $50 \%$ after 20 years. For OW-osteotomy, the probability of survival was $90 \%$ after 10 years. Hui et al. found in their retrospective study of $455 \mathrm{CW}$-osteotomies a probability of survival of $79 \%$ after 10 years and $56 \%$ at 15 years, which is comparable with our results [14]. 
When "being in need for a UKA or TKA" according to the OARSI criteria in addition to joint replacement [9] was considered as end-point, no difference in survival between the CW- and the OW-groups was found. So it seems that patients with a CW-HTO were converted earlier than patients with an OW-HTO. There is no existing literature to compare this result.

Some limitations of our study need to be addressed. Adverse events were measured retrospectively with medical record screening. This could lead to an underestimation of the adverse event rate, due to known under-reporting in medical records. For this reason, we have also assessed the adverse events with a self-administered questionnaire. We identified with this additional information more cases of wound infection, deep venous thrombosis, iliac crest pain and sensory palsy of the CPN. The response rate of the questionnaire was $81 \%$, which is high for a study with patients with a follow-up until 20 years. So the results of this study could still be an underestimation of the real adverse event rate; particularly, the number of wound infections, deep venous thrombosis, iliac crest pain and cases of sensory palsy of the CPN could be underestimated. Because of recall bias, all minor adverse events may be under-represented. However, to the best of our knowledge this is the first study in a large group of patients with this approach.

Secondly, the HTO procedures were performed and supervised by different surgeons over the study period in a university teaching hospital. Moreover, during the study period, the OW-technique was introduced. Although all surgeons were experienced, a single surgeon with experience in both techniques would have been preferable to reduce possible operator-dependent variability. Introduction of a new operation technique could lead to an increased risk of adverse events. However, this situation reflects common orthopaedic practice and improves the generalizability of the results.

\section{Conclusion}

OW-HTO was associated with more adverse events than CW-HTO. Hardware was removed more often in patients with CW- than OW-HTO. OW-HTO resulted in a better survival than CW-HTO; however, an equal proportion of patients were in need for prosthesis in both groups.

Acknowledgments The authors thank all participants for completing the questionnaires.

Conflict of interest The authors report no conflict of interest.

Open Access This article is distributed under the terms of the Creative Commons Attribution 4.0 International License (http:// creativecommons.org/licenses/by/4.0/), which permits unrestricted use, distribution, and reproduction in any medium, provided you give appropriate credit to the original author(s) and the source, provide a link to the Creative Commons license, and indicate if changes were made.

\section{References}

1. Brouwer GM, van Tol AW, Bergink AP, Belo JN, Bernsen RM, Reijman M, Pols HA, Bierma-Zeinstra SM (2007) Association between valgus and varus alignment and the development and progression of radiographic osteoarthritis of the knee. Arthritis Rheum 56(4):1204-1211

2. Brouwer RW, Huizinga MR, Duivenvoorden T, van Raaij TM, Verhagen AP, Bierma-Zeinstra SM, Verhaar JA (2014) Osteotomy for treating knee osteoarthritis. Cochrane Database Syst Rev 12:CD004019

3. Collins NJ, Misra D, Felson DT, Crossley KM, Roos EM (2011) Measures of knee function: International Knee Documentation Committee (IKDC) Subjective Knee Evaluation Form, Knee Injury and Osteoarthritis Outcome Score (KOOS), Knee Injury and Osteoarthritis Outcome Score Physical Function Short Form (KOOS-PS), Knee Outcome Survey Activities of Daily Living Scale (KOS-ADL), Lysholm Knee Scoring Scale, Oxford Knee Score (OKS), Western Ontario and McMaster Universities Osteoarthritis Index (WOMAC), Activity Rating Scale (ARS), and Tegner Activity Score (TAS). Arthritis Care Res (Hoboken) 63(Suppl 11):208-228

4. de Groot IB, Favejee MM, Reijman M, Verhaar JA, Terwee CB (2008) The Dutch version of the Knee injury and Osteoarthritis Outcome Score: a validation study. Health Qual Life Outcomes 6:16

5. Duivenvoorden T, Brouwer RW, Baan A, Bos PK, Reijman M, Bierma-Zeinstra SM, Verhaar JA (2014) Comparison of closingwedge and opening-wedge high tibial osteotomy for medial osteoarthritis of the knee. A randomized controlled trial with a six-year follow-up. J Bone Joint Surg Am 96(17):1425-1432

6. Efe T, Ahmed G, Heyse TJ, Boudriot U, Timmesfeld N, FuchsWinkelmann S, Ishaque B, Lakemeier S, Schofer MD (2011) Closing-wedge high tibial osteotomy: survival and risk factor analysis at long-term follow up. BMC Musculoskelet Disord 12:46

7. Floerkemeier S, Staubli AE, Schroeter S, Goldhahn S, Lobenhoffer P (2013) Outcome after high tibial open-wedge osteotomy: a retrospective evaluation of 533 patients. Knee Surg Sports Traumatol Arthrosc 21(1):170-180

8. Gaasbeek RD, Nicolaas L, Rijnberg WJ, van Loon CJ, van Kampen A (2010) Correction accuracy and collateral laxity in open versus closed wedge high tibial osteotomy. A one-year randomised controlled study. Int Orthop 34(2):201-207

9. Gossec L, Paternotte S, Bingham CO III, Clegg DO, Coste P, Conaghan PG, Davis AM, Giacovelli G et al (2011) OARSI/ OMERACT initiative to define states of severity and indication for joint replacement in hip and knee osteoarthritis. An OMERACT 10 Special Interest Group. J Rheumatol 38(8):1765-1769

10. Hawker GA, Davis AM, French MR, Cibere J, Jordan JM, March L, Suarez-Almazor M, Katz JN, Dieppe P (2008) Development and preliminary psychometric testing of a new OA pain measure-an OARSI/OMERACT initiative. Osteoarthr Cartil 16(4):409-414

11. Hoell S, Suttmoeller J, Stoll V, Fuchs S, Gosheger G (2005) The high tibial osteotomy, open versus closed wedge, a comparison of methods in 108 patients. Arch Orthop Trauma Surg 125(9):638-643 
12. Hui C, Salmon LJ, Kok A, Williams HA, Hockers N, van der Tempel WM, Chana R, Pinczewski LA (2011) Long-term survival of high tibial osteotomy for medial compartment osteoarthritis of the knee. Am J Sports Med 39(1):64-70

13. Martin R, Birmingham TB, Willits K, Litchfield R, Lebel ME, Giffin JR (2014) Adverse event rates and classifications in medial opening wedge high tibial osteotomy. Am J Sports Med 42(5):1118-1126

14. McNamara I, Birmingham TB, Fowler PJ, Giffin JR (2013) High tibial osteotomy: evolution of research and clinical applications-a Canadian experience. Knee Surg Sports Traumatol Arthrosc 21(1):23-31

15. Miller BS, Downie B, McDonough EB, Wojtys EM (2009) Complications after medial opening wedge high tibial osteotomy. Arthroscopy 25(6):639-646

16. Roos EM, Roos HP, Lohmander LS, Ekdahl C, Beynnon BD (1998) Knee Injury and Osteoarthritis Outcome Score (KOOS)development of a self-administered outcome measure. J Orthop Sports Phys Ther 28(2):88-96

17. Sharma L, Song J, Felson DT, Cahue S, Shamiyeh E, Dunlop DD (2001) The role of knee alignment in disease progression and functional decline in knee osteoarthritis. JAMA 286(2):188-195
18. Song EK, Seon JK, Park SJ, Jeong MS (2010) The complications of high tibial osteotomy: closing- versus opening-wedge methods. J Bone Joint Surg Br 92(9):1245-1252

19. van den Bekerom MP, Patt TW, Kleinhout MY, van der Vis HM, Albers GH (2008) Early complications after high tibial osteotomy: a comparison of two techniques. J Knee Surg 21(1):68-74

20. van Egmond N, van Grinsven S, van Loon CJ, Gaasbeek RD, van Kampen A (2014) Better clinical results after closed-compared to open-wedge high tibial osteotomy in patients with medial knee osteoarthritis and varus leg alignment. Knee Surg Sports Traumatol Arthrosc. doi:10.1007/s00167-014-3303-Z

21. van Raaij T, Reijman M, Brouwer RW, Jakma TS, Verhaar JN (2008) Survival of closing-wedge high tibial osteotomy: good outcome in men with low-grade osteoarthritis after 10-16 years. Acta Orthop 79(2):230-234

22. Zorzi AR, da Silva HG, Muszkat C, Marques LC, Cliquet A Jr, de Miranda JB (2011) Opening-wedge high tibial osteotomy with and without bone graft. Artif Organs 35(3):301-307 